\title{
OTTHO: On the Tip of My THOught
}

\author{
Pierpaolo Basile, Marco de Gemmis, Pasquale Lops, and Giovanni Semeraro \\ Dept. of Computer Science - University of Bari \\ Via E. Orabona, 4 - 70125 Bari - Italia \\ \{basilepp, degemmis, lops, semeraro\}@di.uniba.it
}

\begin{abstract}
This paper describes OTTHO (On the Tip of my THOught), a system designed for solving a language game called Guillotine. The rule of the game is simple: the player observes five words, generally unrelated to each other, and in one minute she has to provide a sixth word, semantically connected to the others. The system exploits several knowledge sources, such as a dictionary, a set of proverbs, and Wikipedia to realize a knowledge infusion process. The main motivation for designing an artificial player for Guillotine is the challenge of providing the machine with the cultural and linguistic background knowledge which makes it similar to a human being, with the ability of interpreting natural language documents and reasoning on their content. Our feeling is that the approach presented in this work has a great potential for other more practical applications besides solving a language game.
\end{abstract}

\section{Background and Motivation}

Words are popular features of many games, and they play a central role in many language games. A language game is defined as a game involving natural language in which word meanings play an important role. Language games draw their challenge and excitement from the richness and ambiguity of natural language. In this paper we present a system that tries to play the Guillotine game. The Guillotine is a language game played in a show on RAI, the Italian National Broadcasting Service, in which a player is given a set of five words (clues), each linked in some way to a specific word that represents the unique solution of the game. She receives one word at a time, and must choose between two different proposed words: one is correct, the other one is wrong. Each time she chooses the wrong word, the prize money is divided by half (the reason for the name Guillotine). The five words are generally unrelated to each other, but each of them is strongly related to the word representing the solution. Once the five clues are given, the player has one minute to provide the solution. Often the solution is not so intuitive and the player needs different knowledge sources to reason and find the correct word.

The literature classifies games related to the language in two main categories: 1) word games and 2) language games. Word games do not involve true language, because word meanings are not important. A typical example of word game is Scrabble, in which players take turn placing letters in a grid to form words. 
On the other side, language games, such as crosswords, strongly involve natural language, since word meanings play an important role. WebCrow [1] is the first solver for Italian crosswords and the first system that tackles a language game using the Web as knowledge base. OTTHO (On the Tip of my THOught) is the system we designed to solve the final stage of the Guillotine game. We assume that the five words are provided at the same time, neglecting the initial phase of choosing the words, that only concerns the reduction of the initial prize. With respect to other language games, our "questions" or "clues" are single words, and the answer is a single word. Co-occurrences of terms, providing the evidence of a strong relationship between words, is the key factor for finding a set of candidate words that likely contains the solution. Our system exploits different knowledge sources, such as a dictionary, an encyclopedia, a list of proverbs, etc., as described in the next section.

\section{2 ОТТНО}

Guillotine is a cultural and linguistic game. Therefore, we need to define an extended knowledge base for representing the both the cultural and linguistic background knowledge of the player. After a deep analysis of the correlation between the clues and the solution, we chose to include the following knowledge sources, ranked according to the frequency with which they were helpful in finding the solution of the game:

1) Dictionary 1 : the word representing the solution is contained in the description of a lemma or in some example phrases using that lemma;

2) Encyclopedia 2: as for the dictionary, the description of an article contains the solution, but in this case it is necessary to process a more detailed description of information;

3) Proverbs and aphorisms: short pieces of text in which the solution is found very close to the clues.

In order to exploit these types of sources, it is necessary to organize data gathered from different sources of the same type, to process that information in order to extract and model relationships between words, and to define a reasoning mechanism that, given the clues, is able to select the correct solution of the game among a set of candidate words.

The above mentioned types of sources have different characteristics, therefore different heuristics should be used for building the model. Another important aspect is to define a uniform representation of that model. We decided to use a term-term matrix containing terms occurring in the modeled knowledge source; each cell of the matrix contains the weight representing the degree of correlation between the term on the row and the one on the column. The computation of

${ }^{1}$ De Mauro Italian Dictionary: http://old.demauroparavia.it/

${ }^{2}$ Italian Wikipedia: it.wikipedia.org

${ }^{3}$ Italian proverbs: web.tiscali.it/proverbiitaliani 
the weights is different for each type of knowledge source and takes into account several parameters, as described thoroughly in [2]. Natural language processing techniques adopted for modeling the knowledge sources do not depend on the language used, therefore OTTHO is language independent.

Modeling several knowledge sources realizes a sort of "knowledge infusion" process into the system, in order to create a memory of world facts and linguistic knowledge. An algorithm for retrieving the most appropriate pieces of knowledge associated with the clues is needed to replicate the cognitive mechanism of a human being in the most faithful way. We adopt a spreading activation model [3], which consists of a network data structure of nodes interconnected by links, that may be labeled and/or weighted and usually have directions. The processing is initiated by labeling a set of source nodes with activation weights and proceeds by iteratively propagating that activation to other nodes linked to the source nodes. For each iteration, a termination condition is checked in order to end the search process over the network. We decided to adopt this model as reasoning mechanism of OTTHO. In the network for Guillotine, nodes represent words, while links denote associations between words, obtained from the knowledge sources. The spreading activation is triggered by words given as clues. The activation of clues causes words with related meaning (as modeled in the sources) to become active. At the end of the weight propagation process, the most "active" words represent good candidates to be the solution of the game.

\section{Into the Game}

Our idea is to build a system which helps the user to solve the Guillotine game. OTTHO is able to provide some suggestions in order to make easier finding the correct answer. Figure 1] shows the OTTHO user interface. The clues are visualized on the left of the window, while the suggestions are shown within the text area on the bottom. A timer is displayed on the right of the window, which warns the player on time to provide the answer.

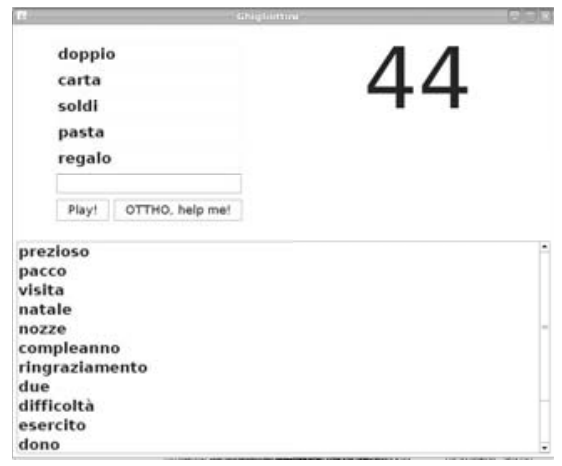

Fig. 1. OTTHO user interface 
As shown in Figure 1 the clues are: doppio (double), carta (paper), soldi (money), pasta and regalo (gift). The solution is pacco (pack), because carta (paper) is a kind of pack (wad), the word pack composes a phrase with both the words gift and double (gift-wrapped package/double pack). Moreover, un pacco di soldi is an Italian expression which means "a lot of money", and pasta can be stored in a package. Notice that the word "pacco" appears in the list of suggestions provided by the system. The accuracy of OTTHO was measured on a dataset of $N=50$ games attempted during the show by human players. A game is solved when the solution occurs in the Candidate Solution List (CSL) produced by OTTHO. Since the component that picks up the unique answer from the CSL is not yet complete, the evaluation is based on the first $k$ words in the CSL (words are ranked according a relevance score computed by the spreading activation model). The accuracy is the percentage of games for which the solution occurs in the CSL. For $k=10$, accuracy is $8 \%$, while for $k=100,40 \%$ of accuracy is achieved. These results are encouraging, given that the average accuracy of the human player on the dataset is about $16 \%$.

\section{Conclusions}

We proposed an artificial player for a language game consisting in guessing a hidden word semantically related to five words given as clues. The system could be used also for implementing an alternative paradigm for associative retrieval on collections of text documents [4, in which an initial indexing phase of documents can spread further "hidden" terms for retrieving other related documents. The identification of hidden terms might rely on the integration of specific pieces of knowledge relevant for the domain of interest. This might represent a valuable strategy for several domains, such as search engine advertising, in which customers' search terms need to be matched with those of advertisers. Spreading activation can be also combined with document retrieval for semantic desktop search 5 .

\section{References}

1. Ernandes, M., Angelini, G., Gori, M.: WebCrow: A Web-Based System for Crossword Solving. In: Veloso, M.M., Kambhampati, S. (eds.) Proc. $20^{\text {th }}$ Nat. Conf. Artif. Intell., $17^{t h}$ Innov. Appl. Artif. Intell., pp. 1412-1417. AAAI Press/MIT Press (2005)

2. Semeraro, G., Lops, P., Basile, P., de Gemmis, M.: On the Tip of my Thought: Playing the Guillotine Game. In: Boutilier, C. (ed.) IJCAI 2009, Proc. of the 21th Int. Joint Conf. on Artificial Intelligence. Morgan Kaufmann, San Francisco (in press, 2009)

3. Collins, A.M., Loftus, E.F.: A spreading activation theory of semantic processing. Psychological Review 82(6), 407-428 (1975)

4. Crestani, F.: Application of Spreading Activation Techniques in Information Retrieval. Artificial Intelligence 11(6), 453-482 (1997)

5. Schumacher, K., Sintek, M., Sauermann, L.: Combining Fact and Document Retrieval with Spreading Activation for Semantic Desktop Search. In: Bechhofer, S., Hauswirth, M., Hoffmann, J., Koubarakis, M. (eds.) ESWC 2008. LNCS, vol. 5021, pp. 569-583. Springer, Heidelberg (2008) 